\title{
ON A NEW FAMILY OF SYMMETRY CODES AND RELATED NEW FIVE-DESIGNS
}

\author{
BY VERA PLESS
}

Communicated by Wallace Givens, May 15, 1969

For every prime $p \equiv-1$ (3) we define a self-orthogonal $(2 p+2$, $p+1)$ code over GF (3). It can be shown that the group leaving a $(2 p+2, p+1)$ code invariant is $\mathrm{PSL}_{2}(p)$. The minimum weights of the first five codes in the family are determined and lead to new 5-designs.

Let $t, r$, and $n$ be integers with $t \leqq r \leqq n$. A $\lambda ; t-r-n$ design $D$ is a collection of subsets of the $n$ integers, each subset containing $r$ elements, such that any $t$-subset of the $n$ integers is contained in the same number $\lambda$ of subsets in $D$. Some designs, a $1 ; 5-6-12$, a 1 ; $5-8-24$, and a $48 ; 5-12-24$ associated with the Mathieu groups $M_{12}$ and $M_{24}$, have been known for a long time. Recently, [1] and [5], $2 ; 5-6-12$ and $2 ; 5-8-24$ designs have been found. Using coding theory [2] other 5-designs were found for $n=24$ and $n=48$. We have found new 5-designs for $n=36$ and $n=60$ and a number of $r$ 's. Also we found new 5-designs for $n=24$ and $n=48$ which are not equivalent to the ones mentioned above. Two $t$-designs are called equivalent if there is a permutation of the $n$ integers so that the subsets of $D$ go onto subsets in $D$.

Let $V_{2 p+2}$ be a vector space over GF(3) with a fixed, orthonormal basis. We call a subspace of this space an error correcting code. We define a family of codes of $\operatorname{dim}(p+1)$ (referred to as $(2 p+2, p+1)$ codes) by a basis $\left(I, S_{p}\right)$ where $S_{p}$ is given below.

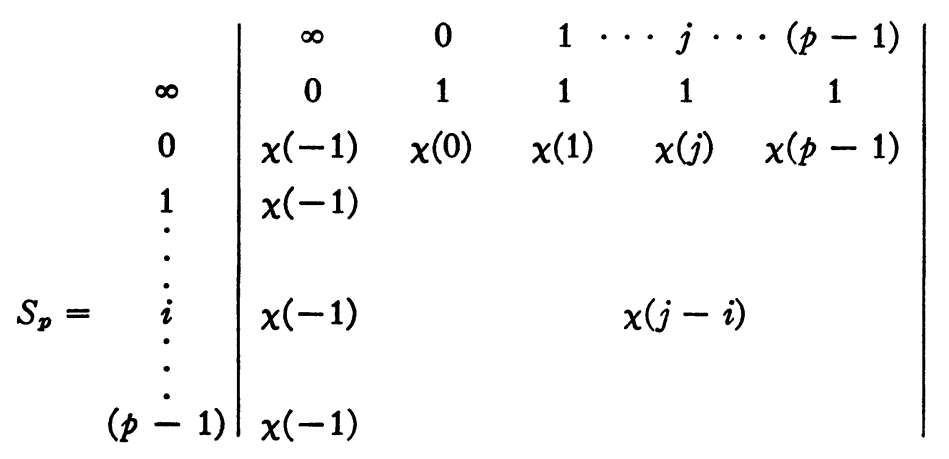

where $\chi(0)=0, \chi($ a square $)=1, \chi($ a nonsquare $)=-1$. We refer to the code generated by $\left(I, S_{p}\right)$ as $C(p)$. 
An important concept in coding is the weight of a vector $v$, this is the number of nonzero components it has. The linear transformations of interest here are the monomial transformations. The matrix of such a transformation has exactly one nonzero element in any row or column. Two codes are said to be equivalent if one is obtained from the other by a monomial transformation. Let $G(p)$ be the group of monomial transformations leaving $C(p)$ invariant.

THEOREM 1. The code $C(p)$ is self orthogonal for all $p$; hence the weight of any vector in $C(p)$ is divisible by 3 .

This follows from the fact that $S_{p}$ is self orthogonal over the reals [4, pp. 209, 210]; hence over $\mathrm{GF}(3)$, and every basis vector is self orthogonal.

THEOREM 2. The group $G(p)$ contains a subgroup isomorphic to $R$ where $R$ modulo $\{I,-I\}$ is isomorphic to $\operatorname{PSL}_{2}(p)$.

Theorem 3. For $p \equiv 1(4),\left(-S_{p}, I\right)$ is also a basis of $C(p)$. For $p \equiv 3(4),\left(S_{p}, I\right)$ is also a basis of $C(p)$.

In general if $\left(I, S_{p}\right)$ is a basis of a code, $\left(-S_{p}^{T}, I\right)$ is the basis of the orthogonal code, which is $C(p)$ again since it is self orthogonal. The result then follows if we note that $S_{p}=S_{p}^{T}$ for $p \equiv 1(4)[4$, p. 210] and $S_{p}=-S_{p}^{T}$ for $p \equiv 3(4)[4$, p. 209].

COROLlaRY. If $p \equiv 1(4), G(p)$ contains a subgroup isomorphic to $Z_{4} R$ where $Z_{4} R=R Z_{4}$ and $R \cap Z_{4}=\{I,-I\}$, and if $p \equiv-1(4), G(p)$ contains a subgroup $Z_{2} \times R$. In both cases $R$ is as in Theorem 2 .

We use this theorem and the following lemma in determining the minimum weights of the first five codes.

LEMma. (a) The weight of the basis vectors of $\left(I, S_{p}\right)$ is $p+1$.

(b) The weight of a linear combination of 2 basis vectors is $(p+7) / 2$.

(c) The weight of a linear combination of 3 basis vectors is $\geqq(p+7) / 2$.

(d) No linear combination of the rows of $S_{p}$ is 0 .

The proof of this lemma depends on the fact that $S_{p} S_{p}^{T}=p I_{p+1}$ over the reals [4, pp. 209,210], $S_{p} S_{p}^{T}=-I_{p+1}$ over GF (3) and hence is nonsingular over GF (3). Part (c) follows from parts (a) and (b).

We relate $t$-designs to codes as in (2). The minimum weight in a code, denoted by $d$, is the weight of the nonzero vector in the code of smallest weight.

Case I. $p=5$. This is a $(12,6)$ code. 
By the use of Theorem 3 and the lemma it can be shown that $d=6$. Hence this code is equivalent to the Golay code (7). It is known that its minimum weight vectors hold $1 ; 5-6-12$ designs (1) and (7).

Case II. $p=11$. This is a $(24,12)$ code:

Again Theorem 3 and the lemma show that $d=9$. Hence by the Assmus-Mattson Theorem (2) the vectors of weights 9, 12, and 15 hold 5-designs. They also hold $4,3,2$, and 1 designs. There is a $(24,12)$ quadratic residue code with the same $d$ as $C(11)$, however, since the entire group of the quadratic residue code (2) and its associated 6; 5-9-24 design (3) is $\mathrm{PSL}_{2}(23)$, and since $C(11)$ is invariant under $\mathrm{PSL}_{2}$ (11) which is not contained in $\mathrm{PSL}_{2}$ (23), this implies that the two codes are not equivalent and the two 5-designs are not equivalent.

Case III. Let $p=17$. This is a $(36,18)$ code.

By Theorem 3 and the lemma we can say that all linear combinations of the basis vectors except 4 or 5 at a time have weight $\geqq 12$. All linear combinations taken 4 at a time were calculated on a computer and found to have weight $\geqq 12$. Again Theorem 3 and the lemma tell us that linear combinations taken 5 at a time have weight $\geqq 12$. Hence $d=12$.

By the Assmus-Mattson Theorem (2), the vectors of weights 12, 15, 18 and 21 hold 5-designs. These are the first 5 -designs found for these parameters. These vectors also hold 4, 3, 2 and 1-designs.

Case IV. Let $p=23$. Here we have a $(48,24)$ code.

It was shown, in part by computer, that $d=15$. Arguing as before, we need only determine linear combinations taken 4,5 , and 6 at a time by computer. Again we have (2) that the vectors of weights 15, 18, 21, 24, and 27 hold 5-designs; also 4, 3, 2 and 1-designs.

There is a $(48,24)$ quadratic residue code with the same $d$ as $C(23)$, (2), however, (2) the entire group of this quadratic residue code and also of its 5-15-48 design (3) is $\mathrm{PSL}_{2}(47)$, and since $C(23)$ is invariant under $\mathrm{PSL}_{2}(23)$ which is not contained in $\mathrm{PSL}_{2}(47)$, this implies that the two codes are not equivalent and their two 5-designs are not equivalent.

Case V. Let $p=29$. Here we have a $(60,30)$ code.

It was shown, in part by computer, that $d=18$.

As before it can be argued that the only linear combinations to be determined on a computer are those taken $4,5,6$, and 7 at a time.

By the Assmus-Mattson theorem again the vectors of weights 18 , $21,24,27,30$, and 33 hold 5-designs; also 4 and lower designs. These are the first 5-designs found for these parameters. 
It should be noted that a $t-r-24\left(t^{\prime}-\mathrm{r}^{\prime}-48\right)$ design associated with $C(11)(C(23))$ has the same $\lambda$ as the $t-r-24\left(t^{\prime}-r^{\prime}-48\right)$ design associated with the quadratic residue $(24,12)((48,24))$ code. This is due to the fact that for these codes the Mac Williams formulas have a unique solution by the theorem in [6].

Note that the five codes above have $d=(p+7) / 2$. This is just equal to the weight of linear combinations of the basis vectors taken 2 at a time. If all the codes of the family were to have this same property, then this would be the first constructive family of codes with $k / n$ and $d / n$ both bounded away from zero. Also the associated 5 -designs would provide the first infinite family of 5-designs.

The computer calculations were shortened by the fact that the matrix $S_{p}$ is invariant under the cyclic shift. I am very grateful to Mrs. Minja Choe for her expert programming. Her acute comments led to a reduction of the number of combinations needed.

I wish to acknowledge helpful discussions with Dr. E. F. Assmus, Jr., Professor A. M. Gleason, Dr. H. F. Mattson, Jr., Mr. John Pierce, and Dr. Richard Turyn.

\section{BIBLIOGRAPHY}

1. E. F. Assmus and H. F. Mattson, Jr., Disjoint Steiner systems associated with the Mathieu groups, Bull. Amer. Math. Soc. 72 (1966), 843-845.

2. —, New 5-designs, J. Combinatorial Theory 6 (1969), 122-151.

3. H. F. Mattson, Private communication.

4. M. Hall, Jr., Combinatorial theory, Blaisdell, Waltham, Mass., 1967.

5. D. R. Hughes, On t-designs and groups, Amer. J. Math. 87 (1965), 761-778.

6. V. Pless, Power moment identities on weight distributions in error correcting codes, Information and Control 6 (1963), 147-152. 215-228.

7. - On the uniqueness of the Golay codes, J. Combinatorial Theory 5 (1968),

Air force Cambridge Research laboratories, L. G. Hanscom field, BEDFORD, MASSACHUSETTS 01730 